\title{
An overview of the role of exclusive enteral nutrition for complicated Crohn's disease
}

\author{
Mustafa Adamji, Andrew S Day \\ Department of Paediatrics, University of Otago Christchurch, Christchurch, New Zealand
}

The role and efficacy of exclusive enteral nutrition (EEN) in the treatment of luminal Crohn's disease (CD) has been well established over the last 2 decades. Consequently, in many centers nutritional therapy is now considered first line therapy in the induction of remission of active CD. However, the use of nutritional therapy in complicated CD has yet to be fully determined. This article aimed to review case reports and clinical trials published in the last decade that have considered and evaluated nutritional therapy in the setting of complicated CD in children and adults. Published literature focusing upon the use of nutritional therapy as part of medical therapy in the management of complicated CD were identified and reviewed. Although there continue to be various interventions utilized for complicated CD, the currently available literature demonstrates that nutritional therapies, especially EEN, have important roles in the management of these complex scenarios. Further assessments, involving large numbers of patients managed with consistent approaches, are required to further substantiate these roles. (Intest Res 2019;17:171-176)

Key Words: Child; Crohn disease; Intestinal fistula; Intestinal obstruction; Nutrition

\section{INTRODUCTION}

Inflammatory bowel disease (IBD) is an umbrella term that describes disorders of unknown etiology that involve chronic inflammation affecting the gut. ${ }^{1}$ Although the peak incidence of IBD is between 15 and 30 years of age, IBD can develop at any age. Up to a quarter of diagnosis are made in the first 2 decades of life. CD is one of the main types of IBD.

CD can affect any part of the gut, from the oral cavity to the anus. ${ }^{1,2} \mathrm{CD}$ typically involves transmural inflammation and can present with features such as abdominal pain, anorexia, tenesmus, and bloody or non-bloody diarrhea. Children most

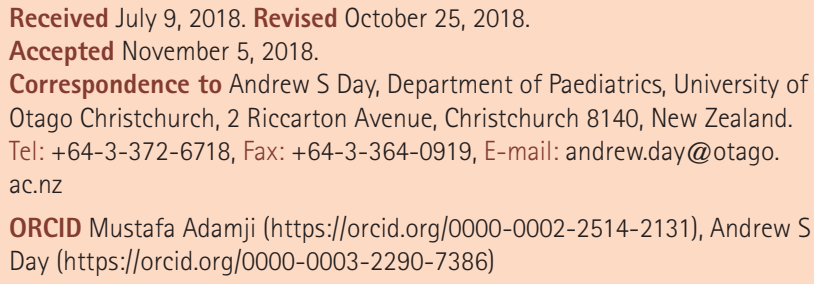

commonly have weight loss, diarrhea and abdominal pain at diagnosis, but can also have unique complications such as growth failure or delayed puberty. CD may also present with extraintestinal symptoms such as arthralgia, skin rashes, oral ulcers, and eye inflammation. Although CD typically commences with luminal inflammation, it can progress to complicated disease featuring strictures or penetration resulting in fistulae and abscesses. Penetrating disease is uncommon at diagnosis in children: 1 large case series showed that 9\% of 276 children had penetrating or structuring disease at diagnosis and $24 \%$ developed 1 or more of these complications within 4 years of diagnosis. $^{2}$

The management of $\mathrm{CD}$ in children involves medications, surgery, nutritional interventions and psychological support, ideally provided within a multi-disciplinary setting. ${ }^{1}$ Medications may be used to induce remission (e.g., corticosteroids or biologics) or to maintain remission (e.g., aminosalicylates and thiopurines). Surgery may be required for disease complications, such as for the management of strictures or for fistuliz- 
ing disease. Nutritional interventions include exclusive enteral nutrition (EEN) to induce remission, ongoing supplementary nutrition to maintain remission (maintenance enteral nutrition, MEN) and general nutritional support to enhance growth. ${ }^{3}$

The benefits of EEN for the induction of remission of active luminal CD have been well described over the last decades with many reports of successful use in children and adults. ${ }^{4,5}$ Consequently, EEN is now recommended in international guidelines as the first line of therapy for children or adolescents with active $\mathrm{CD}^{3,6,7}$ However, EEN may also have roles beyond those described solely for inflammatory luminal CD. This article aims to review recent reports illustrating the use of EEN in complicated CD.

\section{EXCLUSIVE ENTERAL NUTRITION}

EEN involves the use of a nutritionally complete liquid diet given exclusively instead of usual solids and fluids (other than water as required) typically for up to 8 weeks. ${ }^{3}$ Close dietetic support is critical to provide guidance as to the required daily volumes needed to meet the child's individual caloric requirements and to support the child during the period of EEN.

EEN is the initial therapy for induction of remission in almost every child or adolescent with a new diagnosis of CD in New Zealand and many other countries. The local protocol involves the use of EEN for 8 weeks, initially building up to the full required volume of formula over the first 3 to 4 days, depending on tolerance and side effects (as detailed in New Zealand national guidelines available online). ${ }^{8}$ Polymeric formulae (e.g., Ensure; Abbott New Zealand, Auckland, New Zealand or Fortisip; Nutricia New Zealand, Auckland, New Zealand) are preferred over elemental or semi-elemental formulae, due to superior taste characteristics and better tolerance. During EEN, patients would typically be reviewed every 2 weeks or as required. After the completion of the period of EEN, foods are then reintroduced slowly. Supplementary enteral nutrition is typically encouraged when back on normal diet as this may be sufficient to maintain remission. Many children will commence maintenance medical therapy prior to or at the completion of EEN.

Many published reports over the last 2 decades have demonstrated that $\mathrm{EEN}$ is very efficacious for active $\mathrm{CD}$ in children. ${ }^{3,5}$ Generally, EEN induces clinical and biochemical remission in approximately $85 \%$ of patients. Furthermore, a meta-analysis of pediatric studies indicated that EEN had equivalent response to corticosteroids in children with active CD. ${ }^{9}$ Although involv- ing relatively small cohorts, some recent studies confirm and build upon previous data. For instance, a prospective Australian study involving 34 children treated with EEN for 8 weeks demonstrated clinical remission in $84 \%$ and biochemical remission in $76 \%$, while $58 \%$ had early endoscopic response. ${ }^{10} \mathrm{~A}$ subset of this group also had small bowel imaging (magnetic resonance enterography) before and after EEN: 3 of these 14 children had complete transmural healing.

Similarly, $80 \%$ of 40 Spanish children entered remission after 6 to 8 weeks of EEN when evaluated on an intention to treat basis. ${ }^{11}$ When the investigators evaluated the outcomes in the 34 children who had completed the full period of EEN, 32 (92.1\%) were noted to have entered remission.

A retrospective study conducted in the Netherlands assessed the outcomes of EEN in 77 children. ${ }^{12}$ Of the children who completed a course of EEN, $71 \%$ had complete remission whilst a further $26 \%$ had partial remission. The investigators noted that ileal or ileo-colonic disease location at baseline was an important determinant of outcome in this series: $88 \%$ those with ileal or ileocolonic disease entered remission compared to approximately $50 \%$ of those with isolated colonic involvement.

Interestingly, previous pediatric studies have provided conflicting data on the effect of disease location on outcome of EEN. One study conducted in the United Kingdom showed a marked disparity between those children with isolated colonic disease (50\% response rate) and ileal or ileo-colonic disease (remission rate between $92 \%$ and $83 \%$ respectively). ${ }^{13}$ In contrast, a subsequent report from Scotland that included 114 children, showed that those with isolated terminal ileal disease had a lower remission rate, but that location did not otherwise influence outcome. ${ }^{14}$ When evaluated in a Cochrane analysis, there was felt to be insufficient evidence to clearly elucidate the impact of location upon outcome with EEN. ${ }^{15}$

Despite the increasing data on the positive effects of EEN in pediatric $\mathrm{CD}$, there are still limited data on the way in which EEN reduces intestinal inflammation. Traditionally, reduction of antigenic pressure (bowel rest) was considered an important mechanism of EEN. ${ }^{16}$ However, this is unlikely to be the primary factor, as formulae with different protein sources achieve similar remission rates. Recent data suggest that EEN modulates bacterial flora within the gut lumen, thereby likely reducing intestinal inflammation. ${ }^{17,18}$ In addition, active components within formulae used for EEN have direct anti-inflammatory effects on intestinal epithelial cells consequent to downregulation of mucosal pro-inflammatory cytokines. ${ }^{19,20}$ These products also up-regulate various epithelial proteins leading 
to enhanced tight junction function. ${ }^{21}$ Finally, improvement of nutritional status with repletion of nutritional deficiencies is likely to contribute to the some of the benefits seen with EEN. ${ }^{22}$ It is likely that these mechanisms are also relevant for complicated CD.

\section{EEN FOR COMPLICATED CD}

Most reports of the impact and benefits of EEN have focused upon uncomplicated (inflammatory) luminal CD. In recent years an increasing number of publications have demonstrated that EEN is also useful in the setting of complicated CD and extraintestinal $\mathrm{CD}^{23-25}$

\section{EEN in Penetrating CD}

A number of reports have shown that EEN is safe and appears to have beneficial effects in patients with penetrating $\mathrm{CD}^{23-27}$ These cases include isolated fistulizing disease and instances of fistulizing disease complicated by phlegmon development (Table 1).

One of the first reports of the use of EEN in fistulizing disease described a 16-year-old boy with known luminal CD who subsequently developed an enterovesical fistula. ${ }^{23}$ He was initially treated with intravenous antibiotics, bowel rest and total parenteral nutrition for 2 weeks, and then commenced EEN. As he continued to progress well, he was discharged home to continue a further month of EEN, before progressing to normal diet with ongoing supplemental feeds. Eighteen months following diagnosis with the enterovesical fistula, he was in clinical and biochemical remission. Another case report entailed a 13-year-old boy diagnosed with ileocolonic $\mathrm{CD}$ and a perianal fistula. ${ }^{24}$ After the first 4 weeks of EEN (used as sole treatment), he symptomatically improved. After completion of a full 8 weeks of EEN, he was managed with MEN in combination with maintenance drug therapy: he was reported to have no recurrence of perianal disease at 32 months after diagnosis.

Two recent Chinese studies, which included 81 adult patients in total, demonstrated substantial benefits of EEN in the management of fistulizing $\mathrm{CD}^{25,26}$ The first of these reports involved 33 individuals with CD complicated by enterocutaneous or entero-enteric fistula complicated by abscess development. ${ }^{25}$ Evaluations before and after EEN included colonoscopy, biochemical inflammatory markers, and disease activity scores. Twenty-seven of the 31 individuals who completed 12 weeks of EEN entered full remission: only 3 patients required surgery after immediate care. This group of 33 patients included 4 with enterocutaneous fistula: 3 of this group had fistula closure. Furthermore, $76 \%$ of those with enteroenteric fistula with associated abscess had resolution of the abscess. A number of these patients (43\%) also had perianal disease at the time of commencing EEN: the response of perianal disease to EEN was not described.

The second report included 48 subjects with enterocutaneous fistula: all were treated with 12 weeks of EEN. ${ }^{26}$ Successful fistula closure was seen in $62.5 \%$ of these patients. Those with successful response to EEN were more likely to have improved inflammatory and nutritional markers.

These 2 studies included a number of patients with EEN continuing for 12 weeks in duration. Both demonstrated antiinflammatory and nutritional benefits. The outcomes of the first study may be confounded by the use of antibiotics (although the length of antibiotic treatment was not defined). EEN, utilizing a semi-elemental formula, was the sole intervention in the second report.

Finally, a recent case report outlined the role of EEN in the management of 2 children with CD complicated by intra-abdominal abscess or phlegmon with or without associated fistula. ${ }^{27}$ In these 2 cases, EEN was successfully utilized after an initial period of gut rest, antibiotics and total parenteral nutrition. Serial imaging demonstrated successful resolution of the

Table 1. Exclusive Enteral Nutrition in Penetrating CD

\begin{tabular}{llcll}
\hline Reference & Study type & $\begin{array}{c}\text { No. of } \\
\text { patients }\end{array}$ & \multicolumn{1}{c}{ Indication } & \\
\hline 23 & Case report & 1 & Enterovesical & EEN 8 weeks: fistula closure, maintenance of remission 18 months \\
24 & Case report & 1 & Perianal & EEN 8 weeks: fistula resolved, no recurrence over 32 months \\
25 & Case report & 33 & Enteroenteric+abscess, EC & EEN for 12 weeks: $76 \%$ of enteroenteric and abscess healed, 3 of 4 with EC healed \\
26 & Case report & 48 & EC & EEN for 12 weeks: fistula closure in $62.5 \%$ \\
27 & Case report & 2 & Enteroenteric+phlegmon & EEN (8 weeks) with antibiotics: phlegmon resolution \\
\hline
\end{tabular}

These reports involved the use of exclusive enteral nutrition (EEN) in patients with penetrating (fistulizing) CD.

$\mathrm{EC}$, enterocutaneous. 
collection and improved inflammatory markers in both instances. Longer term outcomes were also reported in this case series.

\section{EEN in the Management of Stricturing CD}

A number of reports have noted the roles of EEN may play in the management of CD complicated by stricture development. ${ }^{28.32}$ Although these reports include variable protocols for EEN, different treatment durations and different outcomes, the overall conclusion is that EEN does have a number of benefits in this setting.

Firstly, a prospective observational study described the outcomes of 12 weeks of EEN in 59 patients with stricturing $\mathrm{CD}^{28}$ Fifty of the 59 subjects completed a full course of EEN. Overall, the period of EEN resulted in improved inflammatory markers and clinical symptoms. In addition, nutritional markers improved. While 35 of the subjects were shown to have radiologic remission, more $(\mathrm{n}=48)$ were in symptomatic remission after EEN.

In a retrospective case-control study, 13 of 51 adult patients with stricturing CD managed with preoperative EEN for a mean of 6.3 weeks had stricture resolution and did not require surgery. ${ }^{29}$ Interestingly, the patients managed with EEN who still required surgery had shorter operations, reduced period of time to recommence oral intake postoperatively and fewer surgical complications, suggesting a number of additional benefits of EEN in this context.

A Korean study has also demonstrated improvements in stricturing disease with EEN. ${ }^{30}$ Although most of this group of 66 patients were treated with EEN for uncomplicated luminal $\mathrm{CD}, 10.6 \%$ of the group $(\mathrm{n}=7)$ had strictures. Four of these 7 patients were reported to enter remission with EEN alone and without needing any further escalation of treatment. This subset of patients also remained in remission for the following 2 years while managed with MEN.

A further Chinese study reported the outcomes of EEN in patients with CD complicated by incomplete intestinal obstruction due to stenosis or stricture at various locations (duodenal, jejunal, ileal, ileocolonic or colonic). ${ }^{31}$ The patients received EEN via a nasogastric tube or percutaneous endoscopic gastrostomy/jejunostomy tube for a period of 12 weeks. Seventyfive percent of the cohort underwent remission with clinical and biochemical parameters improving from baseline. Seven of the subjects required surgical intervention during the course of therapy. The longer-term outcomes of this cohort were not described.

Finally, a recent case report described the use of EEN in 1 patient presenting with stricturing duodenal CD on a background of ileal CD. ${ }^{32}$ This patient was initially treated unsuccessfully with an aminosalicylate for 2 weeks. Subsequently, she was treated with 2 weeks of EEN delivered continuously via a nasogastric tube. Symptomatic improvements were seen after this short period of therapy. Repeat endoscopic assessment also documented improvement in the stricture. The report does not, however, quantify the improvement in the stricture. Furthermore, this report does not describe the full duration of EEN or delineate the longer-term outcomes of this patient.

\section{EEN in the Management of Extraintestinal CD}

EEN has also been reported to have roles in patients with CD with orofacial involvement. A single case report noted resolution of oral-facial granulomatosis (OFG) within 2 days of commencing an elemental diet. ${ }^{33}$ More recently, the outcomes of EEN in a group of children with OFG were outlined retrospectively. ${ }^{34}$ Most of the children had luminal CD in addition to their orofacial involvement. Twenty-two children completed 6 weeks of EEN, and 19 showed clinical improvement of the features of OFG. This study indicated there may be a role for EEN as a therapy for both isolated OFG and OFG in conjunction with luminal disease.

The role of EEN in the management of other extraintestinal manifestations of CD has not been formally assessed. Anecdotal reports suggest benefits for the management of erythema nodosum and IBD-associated joint symptoms (unpublished observations, Andrew S Day). Further consideration of the role of $\mathrm{EEN}$ of these aspects of $\mathrm{CD}$ is required.

\section{CONCLUSIONS}

Numerous studies over the last 2 decades have shown that EEN is efficacious and safe as an induction therapy in children with luminal CD. Consequently, this therapy is used in many centers and countries as the initial therapy following diagnosis or subsequently.

However, more recently a number of reports have illustrated that EEN may have roles beyond luminal CD both in children and adults. EEN has been shown to be beneficial in penetrating and stricturing $\mathrm{CD}$, either as an adjunctive therapy or as a bridging therapy delaying or even preventing the need for surgery. However, most of these reports are comprised of small case series or retrospective assessments. More data from prospective trials, and the inclusion of larger numbers of patients 
managed with consistent protocols, are needed to further elucidate the role of EEN in complicated CD and extraintestinal manifestations of CD.

\section{FINANCIAL SUPPORT}

The authors received no financial support for the research, authorship, and/or publication of this article.

\section{CONFLICT OF INTEREST}

No potential conflict of interest relevant to this article was reported.

\section{AUTHOR CONTRIBUTIONS}

Conceptualization: Day AS. Project administration: Day AS. Review of literature: Day AS, Adamji M. Writing - original draft: Adamji M. Writing-review and editing: Adamji M, Day AS. Approval of final manuscript: Adamji M, Day AS.

\section{REFERENCES}

1. Lemberg DA, Day AS. Crohn disease and ulcerative colitis in children: an update for 2014. J Paediatr Child Health 2015;51: 266-270.

2. Van Limbergen J, Russell RK, Drummond HE, et al. Definition of phenotypic characteristics of childhood-onset inflammatory bowel disease. Gastroenterology 2008;135:1114-1122.

3. Critch J, Day AS, Otley A, et al. Use of enteral nutrition for the control of intestinal inflammation in pediatric Crohn disease. J Pediatr Gastroenterol Nutr 2012;54:298-305.

4. Wall CL, Day AS, Gearry RB. Use of exclusive enteral nutrition in adults with Crohn's disease: a review. World J Gastroenterol 2013;19:7652-7660.

5. Day AS, Lopez RN. Exclusive enteral nutrition in children with Crohn's disease. World J Gastroenterol 2015;21:6809-6816.

6. Ruemmele FM, Veres G, Kolho KL, et al. Consensus guidelines of ECCO/ESPGHAN on the medical management of pediatric Crohn's disease. J Crohns Colitis 2014;8:1179-1207.

7. Lochs H, Dejong C, Hammarqvist F, et al. ESPEN guidelines on enteral nutrition: gastroenterology. Clin Nutr 2006;25:260274.

8. Management of inflammatory bowel disease in children and adolescents in New Zealand: a clinical guideline. Starship Web site. https://www.starship.org.nz/for-health-professionals/ new-zealand-child-and-youth-clinical-networks/child-andyouth-clinical-network-for-gastroenterology-services/management-of-inflammatory-bowel-disease. Accessed April 16, 2018.

9. Heuschkel RB, Menache CC, Megerian JT, Baird AE. Enteral nutrition and corticosteroids in the treatment of acute Crohn's disease in children. J Pediatr Gastroenterol Nutr 2000;31:8-15.

10. Grover Z, Muir R, Lewindon P. Exclusive enteral nutrition induces early clinical, mucosal and transmural remission in paediatric Crohn's disease. J Gastroenterol 2014;49:638-645.

11. Navas-López VM, Blasco-Alonso J, Lacasa Maseri S, et al. Exclusive enteral nutrition continues to be first line therapy for pediatric Crohn's disease in the era of biologics. An Pediatr (Barc) 2015;83:47-54.

12. de Bie C, Kindermann A, Escher J. Use of exclusive enteral nutrition in paediatric Crohn's disease in The Netherlands. J Crohns Colitis 2013;7:263-270.

13. Afzal NA, Davies S, Paintin M, et al. Colonic Crohn's disease in children does not respond well to treatment with enteral nutrition if the ileum is not involved. Dig Dis Sci 2005;50:14711475 .

14. Buchanan E, Gaunt WW, Cardigan T, Garrick V, McGrogan P, Russell RK. The use of exclusive enteral nutrition for induction of remission in children with Crohn's disease demonstrates that disease phenotype does not influence clinical remission. Aliment Pharmacol Ther 2009;30:501-507.

15. Zachos M, Tondeur M, Griffiths AM. Enteral nutritional therapy for induction of remission in Crohn's disease. Cochrane Database Syst Rev 2007;(1):CD000542.

16. Heuschkel R. Enteral nutrition should be used to induce remission in childhood Crohn's disease. Dig Dis 2009;27:297305.

17. Kaakoush NO, Day AS, Leach ST, Lemberg DA, Nielsen S, Mitchell HM. Effect of exclusive enteral nutrition on the microbiota of children with newly diagnosed Crohn's disease. Clin Transl Gastroenterol 2015;6:e71.

18. Lewis JD, Chen EZ, Baldassano RN, et al. Inflammation, antibiotics, and diet as environmental stressors of the gut microbiome in pediatric Crohn's disease. Cell Host Microbe 2015; 18:489-500.

19. Fell JM, Paintin M, Arnaud-Battandier F, et al. Mucosal healing and a fall in mucosal pro-inflammatory cytokine mRNA induced by a specific oral polymeric diet in paediatric Crohn's disease. Aliment Pharmacol Ther 2000;14:281-289.

20. de Jong NS, Leach ST, Day AS. Polymeric formula has direct anti-inflammatory effects on enterocytes in an in vitro model 
of intestinal inflammation. Dig Dis Sci 2007;52:2029-2036.

21. Nahidi L, Day AS, Lemberg DA, Leach ST. Differential effects of nutritional and non-nutritional therapies on intestinal barrier function in an in vitro model. J Gastroenterol 2012;47:107117.

22. Gerasimidis K, Talwar D, Duncan A, et al. Impact of exclusive enteral nutrition on body composition and circulating micronutrients in plasma and erythrocytes of children with active Crohn's disease. Inflamm Bowel Dis 2012;18:1672-1681.

23. Sidler MA, Lemberg DA, Day AS. Combination of nutritional therapy and medical therapy for the management of enterovesical fistula in paediatric Crohn's disease: a case report. Clin Nutr ESPEN 2008;3:e89-e91.

24. Wong S, Lemberg DA, Day AS. Exclusive enteral nutrition in the management of perianal Crohn's disease in children. J Dig Dis 2010;11:185-188.

25. Yang Q, Gao X, Chen H, et al. Efficacy of exclusive enteral nutrition in complicated Crohn's disease. Scand J Gastroenterol 2017;52:995-1001

26. Yan D, Ren J, Wang G, Liu S, Li J. Predictors of response to enteral nutrition in abdominal enterocutaneous fistula patients with Crohn's disease. Eur J Clin Nutr 2014;68:959-963.

27. Day AS, Brown SC. The adjunctive role of nutritional therapy in the management of phlegmon in two children with Crohn's disease. Front Pediatr 2017;5:199.

28. Hu D, Ren J, Wang G, et al. Exclusive enteral nutritional therapy can relieve inflammatory bowel stricture in Crohn's disease. J Clin Gastroenterol 2014;48:790-795.

29. Heerasing N, Thompson B, Hendy P, et al. Exclusive enteral nutrition provides an effective bridge to safer interval elective surgery for adults with Crohn's disease. Aliment Pharmacol Ther 2017;45:660-669.

30. Kim HJ, Kim Y, Cho JM, Oh SH, Kim KM. Therapeutic efficacy of oral enteral nutrition in pediatric Crohn's disease: a single center non-comparative retrospective study. Yonsei Med J 2016;57:1185-1191.

31. Xie Y,Zhu WM, Li N, Li JS. Enteral nutritional therapy in Crohn disease complicated with incomplete intestinal obstruction. Zhonghua Wei Chang Wai Ke Za Zhi 2010;13:891-894.

32. Yamamoto T, Nakahigashi M, Umegae S, Kitagawa T, Matsumoto K. Acute duodenal Crohn's disease successfully managed with low-speed elemental diet infusion via nasogastric tube: a case report. World J Gastroenterol 2006;12:649-651.

33. Cameron EA, Middleton SJ. Elemental diet in the treatment of orofacial Crohn's disease. Gut 2003;52:143.

34. Mutalib M, Bezanti K, Elawad M, Kiparissi F. The role of exclusive enteral nutrition in the management of orofacial granulomatosis in children. World J Pediatr 2016;12:421-424. 